\title{
Elementary hypergeometric functions, Heun functions, and moments of MKZ operators
}

\author{
Ana-Maria Acu ${ }^{1 a}$, Ioan Rasa ${ }^{\mathrm{b}}$ \\ ${ }^{a}$ Lucian Blaga University of Sibiu, Department of Mathematics and Informatics, Str. Dr. I. Ratiu, No.5-7, \\ RO-550012 Sibiu, Romania, e-mail: acuana77@yahoo.com \\ ${ }^{b}$ Technical University of Cluj-Napoca, Faculty of Automation and Computer Science, Department of \\ Mathematics, Str. Memorandumului nr.28 Cluj-Napoca, Romania, e-mail: ioan.rasa@math.utcluj.ro
}

\begin{abstract}
We consider some hypergeometric functions and prove that they are elementary functions. Consequently, the second order moments of Meyer-König and Zeller type operators are elementary functions. The higher order moments of these operators are expressed in terms of elementary functions and polylogarithms. Other applications are concerned with the expansion of certain Heun functions in series or finite sums of elementary hypergeometric functions.
\end{abstract}

Keywords: hypergeometric functions, elementary functions, Meyer-König and Zeller type operators, polylogarithms; Heun functions 2010 MSC: 33C05, 33C90, 33E30, 41A36

\section{Introduction}

This paper is devoted to some families of elementary hypergeometric functions, with applications to the moments of Meyer-König and Zeller type operators and to the expansion of certain Heun functions in series or finite sums of elementary hypergeometric functions.

In [4], J.A.H. Alkemade proved that the second order moment of the Meyer-König and Zeller operators can be expressed as

$$
M_{n}\left(e_{2} ; x\right)=x^{2}+\frac{x(1-x)^{2}}{n+1}{ }_{2} F_{1}(1,2 ; n+2 ; x), x \in[0,1) .
$$

Here $e_{r}(t):=t^{r}, t \in[0,1], r \geq 0$, and ${ }_{2} F_{1}(a, b ; c ; x)$ denotes the hypergeometric function.

A closed form of $M_{n}\left(e_{2} ; x\right)$, showing that it is an elementary function, was given in [14]. It is said in [14, p.2]: " The opinion that the second-order moment of the celebrated Meyer-König and Zeller operators is a non-elementary function was tacitly accepted since 1960." The same opinion was firmly expressed in [13, Sect. 2].

This is surprising, because it is elementary to prove that ${ }_{2} F_{1}(1,2 ; n+2 ; x)$ is elementary: such proofs will be given in the next sections. Alternatively, we can use

$$
{ }_{2} F_{1}(1,2 ; 3 ; x)=-2 x^{-2}(x+\log (1-x)),
$$

\footnotetext{
${ }^{1}$ corresponding author
} 
and $[12,2.8(24)]$ :

$$
{ }_{2} F_{1}(a, b ; c+m ; x)=\frac{(c)_{m}(1-x)^{m+c-a-b}}{(c-a)_{m}(c-b)_{m}}\left((1-x)^{a+b-c}{ }_{2} F_{1}(a, b ; c ; x)\right)^{(m)},
$$

where $(r)_{m}:=r(r+1) \ldots(r+m-1), m \geq 1$, and $(r)_{0}:=1$.

They lead to

$$
\begin{aligned}
{ }_{2} F_{1}(1,2 ; n+2 ; x) & =\frac{(3)_{n-1}(1-x)^{n-1}}{(2)_{n-1}(1)_{n-1}}\left({ }_{2} F_{1}(1,2 ; 3 ; x)\right)^{(n-1)} \\
& =-\frac{n+1}{(n-1) !}(1-x)^{n-1}\left(x^{-1}+x^{-2} \log (1-x)\right)^{(n-1)},
\end{aligned}
$$

which is obviously an elementary function. Thus $M_{n}\left(e_{2} ; x\right)$ given by (1.1) is elementary.

On the other hand, $8,(32)]$ provides a closed form of the (elementary) hypergeometric function ${ }_{2} F_{1}(1, m ; m+2 k+1 ; x)$, where $m \geq 1$ and $k \geq 0$ are integers.

In Section 2 we consider the more general function ${ }_{2} F_{1}(m, n ; p ; x)$ with $m, p$ positive integers, $p \geq m+1$, and $n \in \mathbb{R}$. We present a closed form of this function, showing that it is elementary. A general formula for ${ }_{2} F_{1}(a, b ; a+b+l ; x)$ can be found in $[12,2.3(2)]$, but for arbitrary parameters it is not always an elementary function. Generalizing [8, (32)], we give in Section 3 two closed forms for the elementary function ${ }_{2} F_{1}(1, m ; m+l+1 ; x)$ with $m \geq 1, l \geq 0$ integers. Three closed forms for ${ }_{2} F_{1}(1,2 ; n+2 ; x)$ are presented in Section 4. Using a result of [2] we give in Section 5 another proof that the second MKZ moment is elementary. The same result of [2] is instrumental in Section 6, where we show that the higher order MKZ moments can be expressed as finite sums of functions, but containing polylogarithms. In the final section we consider two modifications of the MKZ operators and show that their second order moments are elementary functions; here we use Theorem 2.1 and the fact that ${ }_{2} F_{1}(1,3 ; n+3 ; x)$ is elementary.

Let us mention that the moments of the MKZ operators are involved in studying the asymptotic behavior of the iterates of these operators. For a qualitative result, see [13]. Generalized qualitative and quantitative results can be found in [23, Sect. 2], 7] and [6, Sect.1.3].

Section 8 is devoted to some applications involving Heun equations. The power-series solutions of such an equation are governed by three-term recurrence relations between the successive coefficients, instead of two-term recurrence relations which appear in the hypergeometric case. There are few Heun equations for which the exact solutions are known in terms of simpler functions. This is why expansions of the solutions of Heun equations using other functions instead of powers were considered by many authors; see [16], [17], 19], [24], and the references therein. In particular, expansions in terms of hypergeometric functions and conditions for deriving finite-sum solutions can be found in [16].

Using results from [16], we describe a family of Heun equations for which the solutions are expanded in series of elementary hypergeometric functions. Moreover, we present conditions under which such a series terminates and so the solutions are elementary functions. Other results and applications will be given in a forthcoming paper. 


\section{The function ${ }_{2} F_{1}(m, n ; p ; x)$}

Theorem 2.1. Let $m, p$ be positive integers, $p \geq m+1$, and $n \in \mathbb{R}$. Then

$$
\begin{aligned}
{ }_{2} F_{1}(m, n ; p ; x) & =\frac{(m)_{p-m} x^{1-p}}{(p-m-1) !} \sum_{k=0}^{m-1}(-1)^{k}\left(\begin{array}{c}
m-1 \\
k
\end{array}\right) \\
& \cdot \sum_{j=0}^{p-m-1}\left(\begin{array}{c}
p-m-1 \\
j
\end{array}\right)(-1)^{j} x^{p-m-1-j} \sum_{i=0}^{j}\left(\begin{array}{l}
j \\
i
\end{array}\right)(-1)^{i} \int_{1-x}^{1} s^{i+k-n} d s .
\end{aligned}
$$

Consequently, ${ }_{2} F_{1}(m, n ; p ; x)$ is an elementary function.

Proof. According to the definition,

$$
{ }_{2} F_{1}(m, n ; p ; x)=\sum_{j=0}^{\infty} \frac{(m)_{j}(n)_{j}}{(p)_{j} j !} x^{j} .
$$

This implies

$$
\begin{aligned}
\left(x^{p-1}{ }_{2} F_{1}(m, n ; p ; x)\right)^{(p-m)} & =\sum_{j=0}^{\infty} \frac{(m)_{j}}{(p)_{j}}\left(\begin{array}{c}
n+j-1 \\
j
\end{array}\right)\left(x^{j+p-1}\right)^{(p-m)} \\
& =(m)_{p-m} x^{m-1} \sum_{j=0}^{\infty}\left(\begin{array}{c}
n+j-1 \\
j
\end{array}\right) x^{j}=(m)_{p-m} \frac{x^{m-1}}{(1-x)^{n}} .
\end{aligned}
$$

For the function $x^{p-1}{ }_{2} F_{1}(m, n ; p ; x)$, the Taylor polynomial of degree $p-2 \geq p-1-m$ at 0 vanishes, and so

$$
\begin{aligned}
{ }_{2} F_{1}(m, n ; p ; x) & =\frac{1}{x^{p-1}} \frac{1}{(p-m-1) !} \int_{0}^{t}(x-t)^{p-m-1}\left(t^{p-1}{ }_{2} F_{1}(m, n ; p ; t)\right)^{(p-m)} d t \\
& =\frac{1}{x^{p-1}} \frac{1}{(p-m-1) !} \int_{0}^{t}(x-t)^{p-m-1}(m) p-m t^{m-1}(1-t)^{-n} d .
\end{aligned}
$$

Therefore,

$$
{ }_{2} F_{1}(m, n ; p ; x)=\frac{1}{x^{p-1}} \frac{(m)_{p-m}}{(p-m-1) !} \sum_{k=0}^{m-1}(-1)^{k}\left(\begin{array}{c}
m-1 \\
k
\end{array}\right) \int_{0}^{x}(x-t)^{p-m-1}(1-t)^{k-n} d t .
$$

Now use the binomial formula for $(x-t)^{p-m-1}$ and set $s:=1-t$ in order to verify that the last integral equals

$$
\sum_{j=0}^{p-m-1}\left(\begin{array}{c}
p-m-1 \\
j
\end{array}\right)(-1)^{j} x^{p-m-1-j} \sum_{i=0}^{j}\left(\begin{array}{l}
j \\
i
\end{array}\right)(-1)^{i} \int_{1-x}^{1} s^{i+k-n} d s .
$$

This concludes the proof of (2.1).

Remark 2.1. The proof of Theorem 2.1 is truly elementary: it uses only the definition of the hypergeometric function and Taylor's formula. Another proof has been recently given in [S]]. 
For $m=1$, Theorem 2.1 yields

Corollary 2.1. Let $p \geq 2$ be an integer, and $n \in \mathbb{R}$. Then

$$
{ }_{2} F_{1}(1, n ; p ; x)=\frac{p-1}{x^{p-1}} \sum_{i=0}^{p-2}\left(\begin{array}{c}
p-2 \\
i
\end{array}\right)(x-1)^{p-2-i} \int_{1-x}^{1} s^{i-n} d s .
$$

Proof. Setting $m=1$ in (2.1), we get

$$
\begin{aligned}
{ }_{2} F_{1}(1, n ; p ; x) & =\frac{p-1}{x^{p-1}} \sum_{j=0}^{p-2}\left(\begin{array}{c}
p-2 \\
j
\end{array}\right)(-1)^{j} x^{p-2-j} \sum_{i=0}^{j}\left(\begin{array}{c}
j \\
i
\end{array}\right)(-1)^{i} \int_{1-x}^{1} s^{i-n} d s \\
& =\frac{p-1}{x^{p-1}} \sum_{i=0}^{p-2}(-1)^{i}\left(\begin{array}{c}
p-2 \\
i
\end{array}\right) \sum_{j=i}^{p-2}\left(\begin{array}{c}
p-2-i \\
j-i
\end{array}\right)(-1)^{j} x^{p-2-j} \int_{1-x}^{1} s^{i-n} d s \\
& =\frac{p-1}{x^{p-1}} \sum_{i=0}^{p-2}\left(\begin{array}{c}
p-2 \\
i
\end{array}\right)(x-1)^{p-2-i} \int_{1-x}^{1} s^{i-n} d s .
\end{aligned}
$$

Remark 2.2. Let $\lambda, \mu$, and $\nu$ be nonnegative integers. Theorem 2.1 may be considered also in analogy to the following result (see [12, p. 69], [11, (23)]):

$$
\begin{aligned}
& { }_{2} F_{1}(\nu+1, \nu+\mu+1 ; \nu+\mu+\lambda+2 ; x) \\
& =\frac{(-1)^{\mu+1}(\nu+\mu+\lambda+1) !}{\lambda ! \nu !(\nu+\mu) !(\mu+\lambda) !} \frac{d^{\nu+\mu}}{d x^{\nu+\mu}}\left((1-x)^{\mu+\lambda} \frac{d^{\lambda}}{d x^{\lambda}}\left(\frac{\log (1-x)}{x}\right)\right) .
\end{aligned}
$$

Setting $m=\nu+1, n=\nu+\mu+1, p=\nu+\mu+\lambda+2$, 2.4) leads to

$$
\begin{aligned}
& { }_{2} F_{1}(m, n ; p ; x) \\
& =(-1)^{n-m+1} \frac{p-1}{(p-2) !}\left(\begin{array}{c}
p-2 \\
n-1
\end{array}\right)\left(\begin{array}{c}
p-2 \\
m-1
\end{array}\right) \frac{d^{n-1}}{d x^{n-1}}\left((1-x)^{p-m-1} \frac{d^{p-n-1}}{d x^{p-n-1}}\left(\frac{\log (1-x)}{x}\right)\right),
\end{aligned}
$$

for all integers $m, n, p$ with $p \geq n+1 \geq m+1 \geq 2$.

\section{The function ${ }_{2} F_{1}(1, m ; m+l+1 ; x)$}

Theorem 3.1. Let $m \geq 1$ and $l \geq 0$ be integers. Then

$$
\begin{aligned}
{ }_{2} F_{1}(1, m ; m+l+1 ; x) & =\frac{(m)_{l+1}}{x^{m+l}} \frac{(-1)^{l+1}}{l !}(1-x)^{l} \log (1-x) \\
& +\frac{m+l}{x^{m+l}} \sum_{i=0, i \neq m-1}^{m+l-1}\left(\begin{array}{c}
m+l-1 \\
i
\end{array}\right) \frac{(-1)^{m+l-1-i}}{i-m+1}\left((1-x)^{m+l-1-i}-(1-x)^{l}\right),
\end{aligned}
$$

and also

$$
\begin{aligned}
{ }_{2} F_{1}(1, m ; m+l+1 ; x) & =\frac{(m)_{l+1}}{x^{m+l}} \frac{(-1)^{l+1}}{l !}(1-x)^{l} \log (1-x) \\
& +\frac{(m)_{l+1}}{x^{m+l}}\left\{\frac{(-1)^{l+1}}{l !} \sum_{j=1}^{l} x^{j} \sum_{i=0}^{j-1} \frac{(-1)^{i}}{j-i}\left(\begin{array}{l}
l \\
i
\end{array}\right)-\sum_{i=0}^{m-2} \frac{x^{l+i+1}}{(i+1)_{l+1}}\right\} .
\end{aligned}
$$


Proof. (3.1) is a consequence of (2.3).

To prove (3.2), let us replace in (2.2) $m$ by $1, n$ by $m$, an $p$ by $m+l+1$; thus we have

$$
\left(x_{2}^{n+l} F_{1}(1, m ; m+l+1 ; x)\right)^{(l+1)}=(m)_{l+1} \frac{x^{m-1}}{1-x}=(m)_{l+1}\left(\frac{1}{1-x}-1-x-\cdots-x^{m-2}\right) .
$$

It follows that

$$
x_{2}^{m+l} F_{1}(1, m ; m+l+1 ; x)=(m)_{l+1}\left\{(-1)^{l+1} \frac{(1-x)^{l}}{l !} \log (1-x)-\sum_{i=0}^{m-2} \frac{x^{l+1+i}}{(i+1)_{l+1}}-Q_{l}(x)\right\},
$$

where $Q_{l}$ is a polynomial of degree less or equal to $l$.

The right hand side should be divisible by $x^{m+l}$, hence $Q_{l}(x)$ is the Taylor polynomial of degree $l$, at 0 , for the function $(-1)^{l+1} \frac{(1-x)^{l}}{l !} \log (1-x)$. It is not difficult to obtain

$$
Q_{l}(x)=\frac{(-1)^{l}}{l !} \sum_{j=1}^{l} x^{j} \sum_{i=0}^{j-1} \frac{(-1)^{i}}{j-i}\left(\begin{array}{l}
l \\
i
\end{array}\right)
$$

Putting all things together we get (3.2).

Remark 3.1. Equations (3.1) and (3.2) extend Eq. (32) in [8].

\section{The function ${ }_{2} F_{1}(1,2 ; n+2 ; x)$}

For this function, which is involved in the representation (1.1), we have three explicit expressions:

Theorem 4.1. Let $n \geq 1$ be an integer. Then

$$
\begin{aligned}
{ }_{2} F_{1}(1,2 ; n+2 ; x) & =(-1)^{n} \frac{n+1}{x^{n+1}}\left\{(1-x)^{n-1}[x+n \log (1-x)]-\sum_{j=1}^{n-1}(-1)^{j} \frac{n-j}{j} x^{j}(1-x)^{n-j-1}\right\}, \\
{ }_{2} F_{1}(1,2 ; n+2 ; x) & =(-1)^{n} \frac{n+1}{x^{n+1}}\left\{(1-x)^{n-1}[x+n \log (1-x)]\right. \\
& \left.+\sum_{i=2}^{n} \frac{(-1)^{i}}{i-1}\left(\begin{array}{c}
n \\
i
\end{array}\right)\left[(1-x)^{n-i}-(1-x)^{n-1}\right]\right\}, \\
{ }_{2} F_{1}(1,2 ; n+2 ; x) & =(-1)^{n} \frac{n(n+1)}{x^{n+1}}\left\{(1-x)^{n-1} \log (1-x)+\sum_{j=1}^{n-1} x^{j} \sum_{i=0}^{j-1} \frac{(-1)^{i}}{j-i}\left(\begin{array}{c}
n-1 \\
i
\end{array}\right)\right\}-\frac{n+1}{x} .
\end{aligned}
$$

Proof. (4.1) can be obtained if we continue the calculation in (1.4). (4.2) is a consequence of (3.1), and (4.3) is obtained from (3.2).

Remark 4.1. Other expressions for ${ }_{2} F_{1}(1, m ; m+l+1 ; x)$ and ${ }_{2} F_{1}(1,2 ; n+2 ; x)$ can be obtained from (2.5). 


\section{The second MKZ moment is elementary: another proof}

From [2, (6) and (7)] we know that

$$
M_{n} e_{r}(x)=1+(1-x)^{n+1} \sum_{j=1}^{r}\left(\begin{array}{l}
r \\
j
\end{array}\right) \frac{(-n)^{j}}{(j-1) !} \int_{0}^{\infty} \frac{t^{j-1} e^{-n t}}{\left(1-x e^{-t}\right)^{n+1}} d t .
$$

On the other hand,

$$
\begin{aligned}
\int_{0}^{\infty} t^{j-1} e^{-n t}\left(1-x e^{-t}\right)^{-n-1} d t & =\int_{0}^{\infty} t^{j-1} e^{-n t} \sum_{k=0}^{\infty}\left(\begin{array}{c}
-n-1 \\
k
\end{array}\right)(-1)^{k} x^{k} e^{-k t} d t \\
& =\sum_{k=0}^{\infty}\left(\begin{array}{c}
n+k \\
k
\end{array}\right) x^{k} \int_{0}^{\infty} t^{j-1} e^{-(n+k) t} d t=\sum_{k=0}^{\infty}\left(\begin{array}{c}
n+k \\
k
\end{array}\right) x^{k} \frac{\Gamma(j)}{(n+k)^{j}},
\end{aligned}
$$

so that

$$
M_{n} e_{r}(x)=1+(1-x)^{n+1} \sum_{j=1}^{r}\left(\begin{array}{l}
r \\
j
\end{array}\right)(-n)^{j} f_{n, j}(x),
$$

where

$$
f_{n, j}(x):=\sum_{k=0}^{\infty}\left(\begin{array}{c}
n+k \\
k
\end{array}\right) \frac{1}{(n+k)^{j}} x^{k}, n \geq 1, j \geq 0 .
$$

Let us remark that

$$
f_{n, j}(0)=\frac{1}{n^{j}}
$$

Moreover,

$$
\left(x^{n} f_{n, j}(x)\right)^{\prime}=\sum_{k=0}^{\infty}\left(\begin{array}{c}
n+k \\
k
\end{array}\right) \frac{1}{(n+k)^{j-1}} x^{n-1+k}=x^{n-1} f_{n, j-1},
$$

and so

$$
f_{n, j}(x)=\frac{1}{x^{n}} \int_{0}^{x} t^{n-1} f_{n, j-1}(t) d t, j \geq 1 .
$$

We have $f_{n, 0}(x)=\frac{1}{(1-x)^{n+1}}$, and (5.4) yields

$$
f_{n, 1}(x)=\frac{1}{n(1-x)^{n}} .
$$

From (5.4) and (5.5) it is easy to obtain

$$
f_{n, 2}(x)=\frac{(-1)^{n-1}}{n x^{n}}\left\{\sum_{i=1}^{n-1}\left(\begin{array}{c}
n-1 \\
i
\end{array}\right) \frac{(-1)^{i}}{i} \frac{1-(1-x)^{i}}{(1-x)^{i}}-\log (1-x)\right\} .
$$

Now (5.1), (5.5) and (5.6) show that $M_{n} e_{2}(x)$ is an elementary function. 


\section{Higher order moments of the MKZ operators}

We recall the definition (see [20]) of the dilogarithm

$$
L i_{2}(x):=-\int_{0}^{x} \frac{\log (1-t)}{t} d t=\sum_{k=1}^{\infty} \frac{x^{k}}{k^{2}},|x| \leq 1,
$$

and the polylogarithm of order $n$,

$$
L i_{n}(x):=\int_{0}^{x} \frac{L i_{n-1}(t)}{t} d t=\sum_{k=1}^{\infty} \frac{x^{k}}{k^{n}}, n \geq 3 .
$$

Using (5.4) and (5.6) we get

$$
\begin{aligned}
f_{n, 3}(x) & =\frac{(-1)^{n-1}}{n x^{n}} \int_{0}^{x}\left\{\sum_{i=1}^{n-1}\left(\begin{array}{c}
n-1 \\
i
\end{array}\right) \frac{(-1)^{i}}{i} \frac{1-(1-t)^{i}}{t(1-t)^{i}}-\frac{\log (1-t)}{t}\right\} d t \\
& =\frac{(-1)^{n-1}}{n x^{n}} \int_{0}^{x}\left\{\sum_{i=1}^{n-1}\left(\begin{array}{c}
n-1 \\
i
\end{array}\right) \frac{(-1)^{i}}{i}\left(\sum_{l=0}^{i-2}(1-t)^{l-i}+\frac{1}{1-t}\right)-\frac{\log (1-t)}{t}\right\} d t
\end{aligned}
$$

so that finally,

$$
f_{n, 3}(x)=\frac{(-1)^{n-1}}{n x^{n}}\left\{\sum_{i=1}^{n-1}\left(\begin{array}{c}
n-1 \\
i
\end{array}\right) \frac{(-1)^{i}}{i}\left[\sum_{l=0}^{i-2} \frac{1-(1-x)^{i-l-1}}{(i-l-1)(1-x)^{i-l-1}}-\log (1-x)\right]+L i_{2}(x)\right\} .
$$

Theorem 6.1. Let $n \geq 2$. For each $j \geq 2$, the functions $x^{n} f_{n, j}(x)$ are linear combinations, with constant coefficients, of $n$ functions as follows:

1) For $2 \leq j \leq n$ :

$$
\frac{1-(1-x)^{i}}{(1-x)^{i}}, i=1, \ldots, n-j+1 ; \log (1-x) ; L i_{2}(x), \ldots, L i_{j-1}(x) .
$$

2) For $j=n+1$ :

$$
\log (1-x) ; L i_{2}(x), \ldots, L i_{n}(x)
$$

3) For $j \geq n+2$ :

$$
L i_{j-n}(x), \ldots, L i_{j-1}(x) .
$$

Proof. We use induction on $j$. The first step, involving $j=2, j=3$, is accomplished by using (5.6) and (6.3). Then the induction is continued with the recurrence relation (5.4) and (6.1), 6.2).

Remark 6.1. Using (5.1) and Theorem 6.1 we get information about the structure of the moments $M_{n} e_{r}$. In particular, we see once more that $M_{n} e_{2}$ is elementary. The higher order moments contain polylogarithms. 


\section{Other operators}

Let $m_{n, k}(x):=\left(\begin{array}{c}n+k \\ k\end{array}\right)(1-x)^{n+1} x^{k}, x \in[0,1]$, and for $f \in L_{1}[0,1]$

$$
L_{n}(f ; x):=\sum_{k=0}^{\infty} \frac{(n+k+1)(n+k+2)}{n+1}\left(\int_{0}^{1} m_{n, k}(t) f(t) d t\right) m_{n, k}(x) .
$$

The operators $L_{n}$ were investigated in [10]; they coincide with the operators $M_{n, 2}$ from [15]; details can be found in [9].

It was proved in [10] (see also [9, p. 123]) that

$$
L_{n}\left(e_{2} ; x\right)=x^{2}+\frac{2 x(1-x)^{2}}{n+2}{ }_{2} F_{1}(1,3 ; n+3 ; x) .
$$

Closed forms of the elementary functions ${ }_{2} F_{1}(1,3 ; n+3 ; x)$ can be obtained from (3.1) and (3.2) taking $m=3, l=n-1$.

In particular, we see that $L_{n}\left(e_{2} ; x\right)$ is an elementary function. It would be interesting to study from this point of view the higher order moments of $L_{n}$ and, more generally, of other modifications of the MKZ operators presented in [9].

Remark 7.1. In [5, Chapter 7] the generalized Meyer-König and Zeller operators are defined as follows:

$$
\begin{gathered}
\left(M_{n, r}^{\alpha, \beta} f\right)(x):=(1-x)^{n+r} \sum_{k=0}^{\infty}\left(\begin{array}{c}
n+r+k-1 \\
k
\end{array}\right) x^{k} f\left(\frac{k+\beta}{n+k+\alpha}\right), \\
x \in[0,1), \alpha, \beta \in \mathbb{R}, \alpha \geq \beta \geq 0, r \in \mathbb{Z}, n, n+r \in \mathbb{N},
\end{gathered}
$$

and it is proved that

$$
\left(M_{n, r}^{\alpha, \beta} e_{1}\right)(x)=\frac{\beta}{n+\alpha}{ }_{2}^{2} F_{1}(1, \alpha-r ; n+1+\alpha ; x)+\frac{n+r-\beta}{n+1+\alpha} x_{2} F_{1}(1, \alpha-r+1, n+2+\alpha ; x) .
$$

If $\alpha \geq 0$ is an integer, we can apply Theorem 2.1 to conclude that $M_{n, r}^{\alpha, \beta} e_{1}$ is an elementary function.

Moreover, suppose that $\alpha \geq 0$ is an integer and $r=\alpha+1$. Then (7.2) yields

$$
\left(M_{n, \alpha+1}^{\alpha, \beta} e_{1}\right)(x)=\frac{\beta}{n+\alpha}+\left(1-\frac{\beta}{n+\alpha}\right) x .
$$

Let $g_{j}(x):=(1-x)^{j}, j=0,1, \ldots$ Using a trick invented by Professor Ulrich Abel (see [1]]), we can write

$$
\begin{aligned}
\left(M_{n, \alpha+1}^{\alpha, \beta} g_{j}\right)(x) & =\frac{(n+\alpha-\beta)^{j}}{(n+\alpha) !}(1-x)^{n+\alpha+1} \sum_{k=0}^{\infty} \frac{(n+k+\alpha) !}{k !} \frac{x^{k}}{(n+k+\alpha)^{j}} \\
& =\frac{(n+\alpha-\beta)^{j}}{(n+\alpha) !}(1-x)^{n+\alpha+1}\left(\sum_{k=0}^{\infty} \frac{x^{n+k+\alpha}}{(n+k+\alpha)^{j}}\right)^{(n+\alpha)} \\
& =\frac{(n+\alpha-\beta)^{j}}{(n+\alpha) !}(1-x)^{n+\alpha+1}\left(L i_{j}(x)\right)^{(n+\alpha)} .
\end{aligned}
$$


Combined with $e_{m}=\sum_{j=0}^{m}(-1)^{j}\left(\begin{array}{c}m \\ j\end{array}\right) g_{j}$, this leads to

$$
\left(M_{n, \alpha+1}^{\alpha, \beta} e_{m}\right)(x)=\frac{(1-x)^{n+\alpha+1}}{(n+\alpha) !} \sum_{j=0}^{m}(-1)^{j}\left(\begin{array}{c}
m \\
j
\end{array}\right)(n+\alpha-\beta)^{j}\left(L i_{j}(x)\right)^{(n+\alpha)} .
$$

Now it is easy to recover (7.3) and to see that $M_{n, \alpha+1}^{\alpha, \beta} e_{2}$ is an elementary function.

\section{Elementary Heun functions}

The general Heun equation in canonical form is (see [18], 21], [22]):

$$
\frac{d^{2} u}{d x^{2}}+\left(\frac{\gamma}{x}+\frac{\delta}{x-1}+\frac{\varepsilon}{x-a}\right) \frac{d u}{d x}+\frac{\alpha \beta x-q}{x(x-1)(x-a)} u=0,
$$

where the parameters satisfy $\alpha+\beta+1=\gamma+\delta+\varepsilon$.

Under the hypotheses that $\alpha \beta \neq 0$ and $\gamma+\varepsilon$ is not zero or a negative integer, an expansion of the solution $u(x)$ as a series of hypergeometric functions is investigated in [16].

Let $m$ and $p$ be positive integers, $p \geq m+1$, and $n \in \mathbb{R}, n \neq 0$. In (8.1) choose

$$
\begin{gathered}
\alpha=m, \beta=n, \gamma=p+1-m-n, \delta=m+n-p+1, \\
\varepsilon=m+n-1, a=\frac{1}{2}, q=\frac{1}{2}(m n-(m+n-p)(m+n-1)) .
\end{gathered}
$$

Theorem 8.1. With the above parameters, the solution $u(x)$ of (8.1) is given by

$$
u(x)=\sum_{k=0}^{\infty} \frac{\left(\frac{m+n-1}{2}\right)_{k}\left(\frac{p-m}{2}\right)_{k}\left(\frac{p-n}{2}\right)_{k}}{k !\left(\frac{p}{2}\right)_{k}\left(\frac{p+1}{2}\right)_{k}} F_{1}(m, n ; p+2 k ; x),
$$

and ${ }_{2} F_{1}(m, n ; p+2 k ; x)$ are elementary functions. Moreover, if there exists a positive integer $r$ such that $m+n=3-2 r$ or $n-p=2 r-2$, then the series (8.2) reduces to a finite sum and so $u(x)$ is an elementary function.

Proof. It is easy to check that $\alpha \beta \neq 0, \gamma+\varepsilon=p \geq 2, a=\frac{1}{2}, \gamma+\delta=2, q=a \alpha \beta+a(1-\delta) \varepsilon$, and so the conditions [16, (43)] are satisfied. According to [16, (45)], the solution of the Heun equation (8.1) is given by

$$
u(x)=\sum_{k=0}^{\infty} \frac{\left(\frac{\varepsilon}{2}\right)_{k}\left(\frac{\gamma+\varepsilon-\alpha}{2}\right)_{k}\left(\frac{\gamma+\varepsilon-\beta}{2}\right)_{k}}{k !\left(\frac{\gamma+\varepsilon}{2}\right)_{k}\left(\frac{1+\gamma+\varepsilon}{2}\right)_{k}} F_{1}(\alpha, \beta ; \gamma+\varepsilon+2 k ; x),
$$

which leads immediately to (8.2).

Using Theorem 2.1 we see that ${ }_{2} F_{1}(m, n ; p+2 k ; x)$ is an elementary function for each $k \geq 0$.

Let $m+n=3-2 r$, or $n-p=2 r-2$, with $r$ an integer, $r \geq 1$. Then

$$
\left(\frac{m+n-1}{2}\right)_{r}=0, \text { or }\left(\frac{p-n}{2}\right)_{r}=0
$$


and so the series in (8.2) reduces to the sum for $k \in\{0,1, \ldots, r-1\}$; this means that in this case $u(x)$ is an elementary function for which explicit expressions can be obtained using results from Sections 2,4.

Remark 8.1. Usually, a Heun function is normalized by $u(0)=1$. For the equation (8.1), [16, (46)] yields

$$
u(0)={ }_{3} F_{2}\left(\frac{\gamma+\varepsilon-\alpha}{2}, \frac{\gamma+\varepsilon-\beta}{2}, \frac{\varepsilon}{2} ; \frac{\gamma+\varepsilon}{2}, \frac{1+\gamma+\varepsilon}{2} ; 1\right),
$$

where ${ }_{3} F_{2}$ is Clausen's generalized hypergeometric function. With the parameters used in Theorem 8.1 we get

$$
u(0)={ }_{3} F_{2}\left(\frac{p-m}{2}, \frac{p-n}{2}, \frac{m+n-1}{2} ; \frac{p}{2}, \frac{1+p}{2} ; 1\right) .
$$

Thus $u(x) / u(0)$ satisfies the normalization condition.

Acknowledgement. The work of the first author was financed by Lucian Blaga University of Sibiu \& Hasso Plattner Foundation research grants LBUS-IRG-2019-05.

\section{References}

[1] U. Abel, Elementary function representations for the moments of the Meyer-König and Zeller operators, arXiv: 1911.02563v1 [math.GM]

[2] U. Abel, The moments for the MeyerKönig and Zeller operators, J. Approx. Theory 82(3), 352361 (1995).

[3] U. Abel, Unpublished manuscript, 2019.

[4] J.A.H. Alkemade, The second moment for the MeyerKönig and Zeller operators, J. Approx. Theory 40(3), 261273 (1984).

[5] J.A.H. Alkemade, On a differential equation and the Laplace- Stieltjes transform in the theory of linear positive operators, Thesis, Delft University Press, 1984.

[6] F. Altomare, M. Cappelletti Montano, V. Leonessa, I. Raa, Markov Operators, Positive Semigroups and Approximation Processes, 61, De Gruyter Studies in Mathematics, 2014.

[7] F. Altomare, I. Rasa, Lipschitz contractions, unique ergodicity and asymptotics of Markov semigroups, Bollettino U. M. I. (9), 5(2012), 1-17.

[8] A. Bărar, G. Mocanu, I. Raşa, Heun functions related to entropies, RACSAM (2019), 113: 819-830.

[9] J. Bustamante, M.A. Jiménez Pozo, Meyer-König and Zeller operators and some of their modifications, Jaen J. Approx. 5(2), 2013, 101-178.

[10] W. Chen, The second moment for the Meyer-König and Zeller-type operators, J. Xiamen Univ. Natur. Sci. 29(2), 1990, 119-123 (in Chinese).

[11] J. Detrich, R.W. Conn, Finite sum evaluation of the Gauss hypergeometric function in an important special case, Math. Comput. 33, 788791 (1979). 
[12] A. Erdelyi, Higher Transcendental Functions, Vol. I, McGraw-Hill Book Company, New York, 1953.

[13] I. Gavrea, M. Ivan, On the iterates of positive linear operators preserving the affine functions, J. Math. Anal. Appl. 372 (2010) 366-368.

[14] I. Gavrea, M. Ivan, An elementary function representation of the second-order moment of the Meyer-König and Zeller operators, Mediterr. J. Math. (2018), 15:20.

[15] H. Heilmann, Commutativity of Durrmeyer-type modifications of Meyer-König and Zeller and Baskakov operators, in: B. Bojanov (ed.), Constructive Theory of Functions, Proc. Internat. Conf., Varna, Bulgaria, 2002, pp. 295-301, DARBA, Sofia.

[16] T.A. Ishkhanyan, T.A. Shahverdyan, A.M. Ishkhanyan, Expansions of the solutions of the general Heun equation governed by two-term recurrence relations for coefficients, Advances in High Energy Physics 2018, Article ID 4263678, (2018).

[17] A. Ishkhanyan, K.A. Suominen, New solutions of Heuns general equation, J. Phys. A Math. Gen. 36, L81L85 (2003).

[18] G. Kristensson, Second Order Differential Equations. Special Functions and Their Classification, Springer, 2010.

[19] C. Leroy, A.M. Ishkhanyan, Expansions of the solutions of the confluent Heun equation in terms of the incomplete Beta and the Appell generalized hypergeometric functions, Integral Transform. Spec. Funct. 26(6), 451459 (2015).

[20] L. Lewin, Polylogarithms and associated functions, North-Holland, Amsterdam, 1981.

[21] R.S. Maier, The 192 solutions of the Heun equation, Math. Comp. 76, 811-843 (2007).

[22] R.S. Maier, On reducing the Heun equation to the hypergeometric equation, J. Differ. Equ. $213,171203(2005)$.

[23] I. Rasa, $C_{0}$-Semigroups and iterates of positive linear operators: asymptotic behaviour, Rendiconti del Circolo Matematico di Palermo, Ser. II, Suppl. 82(2010),123-142.

[24] V.A. Shahnazaryan, T.A. Ishkhanyan, T.A. Shahverdyan, A.M. Ishkhanyan, New relations for the derivative of the confluent Heun function, Armen. J. Phys. 5, 146156 (2012). 